\section{TATRA \\ MOUNTaiNS \\ Mathematical Publications}

DOI: $10.2478 / \mathrm{tmmp}-2020-0035$

Tatra Mt. Math. Publ. 77 (2020), 99-108

\title{
ON SOME MULTINOMIAL SUMS RELATED TO THE FIBONACCI TYPE NUMBERS
}

\author{
IwONA WŁOCH ${ }^{1}$ - ANDRZEJ WŁOCH ${ }^{2}$ \\ ${ }^{1}$ Department of Discrete Mathematics, Rzeszow University of Technology, Rzeszow, POLAND \\ ${ }^{2}$ Department of Mathematical Modeling, Rzeszow University of Technology, Rzeszow, POLAND
}

\begin{abstract}
In this paper we investigate Fibonacci type sequences defined by kth order linear recurrence. Based on their companion matrix and its graph interpretation we determine multinomial and binomial formulas for these sequences. Moreover we present a graphical rule for calculating the words of these sequences from the Pascal's triangle.
\end{abstract}

\section{Introduction}

Fibonacci numbers are defined recursively by $F_{n}=F_{n-1}+F_{n-2}$ for $n \geq 2$ with initial conditions $F_{0}=0, F_{1}=1$. Using $F_{n-2}=F_{n}-F_{n-1}$, Fibonacci numbers can be extended to negative integers and $F_{-n}=(-1)^{n+1} F_{n}$. Consequently, the sequence $\left\{F_{n}\right\}$ has the form $\ldots,-8,5,-3,2,-1,1,0,1,1,2,3,5,8, \ldots$ Many generalizations of Fibonacci numbers were studied, see for example the list given in [1]. In 9] $k$-Fibonacci numbers were defined by $g_{n}=g_{n-1}+g_{n-2}+\cdots+g_{n-k}$ for $n \geq k \geq 2$ with $g_{0}=g_{1}=\cdots=g_{k-2}=0$ and $g_{k-1}=1$. Some properties of the sequence $\left\{g_{n}\right\}$ were studied in [9] and next in [8. More general case of sequence $\left\{g_{n}\right\}$ was studied by Kalman, see for details [5]. In [2] Er introduced a family of $k$ sequences of generalized Fibonacci numbers in the following way.

Let $k \geq 2, c_{j}, j \in\{1, \ldots, k\}$ be integers. Then for an integer $1 \leq i \leq k$ generalized Fibonacci numbers $f_{n}^{i}$ are defined as

$$
f_{n}^{i}=\sum_{j=1}^{k} c_{j} f_{n-j}^{i} \quad \text { for } n>0
$$

(C) 2020 Mathematical Institute, Slovak Academy of Sciences.

2010 Mathematics Subject Classification: 11B37, 11B39, $11 \mathrm{~B} 65$.

Keywords: Fibonacci numbers, recurrence, multinomial formulas.

Licensed under the Creative Commons Attribution-NC-ND4.0 International Public License. 


\section{IWONA WŁOCH - ANDRZEJ WŁOCH}

with initial conditions $f_{n}^{i}=\left\{\begin{array}{ll}1 & \text { if } i=1-n, \\ 0 & \text { otherwise, }\end{array}\right.$ for $\quad 1-k \leq n \leq 0$.

The number $f_{n}^{i}$ is the $n$th term of the $i$ th sequence. If $k=2$ and $c_{1}=c_{2}=1$, then $F_{n}=f_{n-1}^{1}=f_{n}^{2}$ for $n \geq 0$.

Based on an approach taken by Kalman [5], Er used for these sequences a $k \times k$ matrix $A$ of the form

$$
A=\left[\begin{array}{cccc}
c_{1} & c_{2} & \ldots & c_{k} \\
1 & 0 & \ldots & 0 \\
\vdots & \ddots & \ddots & \vdots \\
0 & 0 & 1 & 0
\end{array}\right]
$$

and showed that

$$
A^{n}=\left[\begin{array}{cccc}
f_{n}^{1} & f_{n}^{2} & \cdots & f_{n}^{k} \\
f_{n-1}^{1} & f_{n-1}^{2} & \cdots & f_{n-1}^{k} \\
\vdots & \vdots & \ddots & \vdots \\
f_{n-k+1}^{1} & f_{n-k+1}^{2} & \cdots & f_{n-k+1}^{k}
\end{array}\right] .
$$

In this paper, we consider a generalization of Fibonacci numbers which relates to sequences (11).

Let $k \geq 2, c_{i} \geq 0, i \in\{1, \ldots, k\}$ be integers such that there are at least two positive integers $c_{p}, c_{q}$ where $p \neq q$ and $1 \leq p, q \leq k$. Generalized Fibonacci numbers are defined recursively by the $k$ th order linear recurrence relation

$$
f_{n}=c_{1} f_{n-1}+c_{2} f_{n-2}+\cdots+c_{k} f_{n-k} \text { for } n>0
$$

with given nonnegative integers $f_{1-k}, \ldots, f_{-1}, f_{0}$, and there is $1-k \leq j \leq 0$ such that $f_{j}>0$.

For special values of $k, c_{i}$, and $f_{1-i}, i \in\{1, \ldots, k\}$, the formula (3) gives the well-known classical sequences of the Fibonacci type, see the Table 1.

TABLE 1. Generalized Fibonacci numbers for special values $k, c_{i}$ and $f_{i}$.

\begin{tabular}{|l||c|c|c|c|c|c|c||l|}
\hline Sequence & $k$ & $c_{1}$ & $c_{2}$ & $c_{3}$ & -2 & -1 & 0 & Recursion \\
\hline \hline Fibonacci & 2 & 1 & 1 & & & 0 & 1 & $F_{n}=F_{n-1}+F_{n-2}$ for $n \geq 1$ \\
\hline Lucas & 2 & 1 & 1 & & & 2 & 1 & $L_{n}=L_{n-1}+L_{n-2}$ for $n \geq 1$ \\
\hline Pell & 2 & 2 & 1 & & & 0 & 1 & $P_{n}=2 P_{n-1}+P_{n-2}$ for $n \geq 1$ \\
\hline Jacobsthal & 2 & 1 & 2 & & & 0 & 1 & $J_{n}=J_{n-1}+2 J_{n-2}$ for $n \geq 1$ \\
\hline Padovan & 3 & 0 & 1 & 1 & 1 & 1 & 1 & $P v(n)=P v(n-2)+P v(n-3), n \geq 1$ \\
\hline Tribonacci & 3 & 1 & 1 & 1 & 0 & 0 & 1 & $T_{n}=T_{n-1}+T_{n-2}+T_{n-3}$ for $n \geq 1$ \\
\hline
\end{tabular}


Apart from the recurrence relations for Fibonacci type sequences, binomial formulas were determined. We recall some of them:

1. F. E. A. Lucas 7 ]

$$
F_{n-1}=\sum_{i=0}^{\left\lfloor\frac{n-1}{2}\right\rfloor}\left(\begin{array}{c}
n-i-1 \\
i
\end{array}\right),
$$

2. H. W. Gould [10]

$$
L_{n-1}=n \sum_{i=0}^{\left\lfloor\frac{n}{2}\right\rfloor}\left(\begin{array}{c}
n-i \\
i
\end{array}\right) \frac{1}{n-i},
$$

3. A. F. Haradam 4

$$
P_{n-1}=\sum_{i=0}^{\left\lfloor\frac{n}{2}\right\rfloor}\left(\begin{array}{c}
n \\
2 i+1
\end{array}\right) 2^{i},
$$

4. S. Falcon Santana, J. L. Diaz-Barrero [3]

$$
P_{n}=\sum_{i=0}^{\left\lfloor\frac{n}{2}\right\rfloor}\left(\begin{array}{c}
n-i \\
i
\end{array}\right) 2^{n-2 i},
$$

5. E. Kilic, H. Prodinger 6]

$$
P_{n}=\sum_{0 \leq i \leq j \leq n}\left(\begin{array}{c}
n-i \\
j
\end{array}\right)\left(\begin{array}{l}
j \\
i
\end{array}\right),
$$

6. B. Cloitre [10]

$$
J_{n-1}=\sum_{i=0}^{\left\lceil\frac{n}{2}\right\rceil} 2^{i}\left(\begin{array}{c}
n-i \\
i
\end{array}\right),
$$

7. E. Kilic, H. Prodinger [6]

$$
T_{n}=\sum_{0 \leq j \leq i \leq n}\left(\begin{array}{c}
n-i \\
i-j
\end{array}\right)\left(\begin{array}{c}
i-j \\
j
\end{array}\right) .
$$

Other binomial formulas for integer sequences can be found in [10]. Inspired by these results, we looked for a general binomial formula for all sequences defined by the recurrence (3).

The purpose of this paper is to investigate the connections between sequences, matrices and directed multigraphs. This leads to the discovery of a method of deriving multinomial and binomial formulas for these sequences. Obtained formulas allow us to formulate a graphical rule for calculating generalized Fibonacci numbers from the Pascal's triangle. Consequently, we present new binomial formulas for these numbers. 


\section{IWONA WŁOCH - ANDRZEJ WŁOCH}

\section{Main results}

Firstly, we give dependencies between $n$th generalized Fibonacci number $f_{n}$ and $n$th term of the $i$ th sequence.

TheOREM 2.1. Let $k \geq 2$ and $n \geq 1-k$ be integers. Then $f_{n}=\sum_{i=1}^{k} f_{1-i} f_{n}^{i}$.

Proof. (by the induction on $\mathrm{n}$ ) If $1-k \leq n \leq 0$, then from the definition of $f_{n}^{i}$ we obtain

$\sum_{i=1}^{k} f_{1-i} f_{n}^{i}=f_{0} f_{n}^{1}+f_{-1} f_{n}^{2}+\cdots+f_{n} f_{n}^{1-n}+\cdots+f_{1-k} f_{n}^{k}=f_{n} f_{n}^{1-n}=f_{n} \cdot 1=f_{n}$.

Assume that $n>0$ and let $f_{n}=\sum_{i=1}^{k} f_{1-i} f_{n}^{i}$. We shall show that

$$
f_{n+1}=\sum_{i=1}^{k} f_{1-i} f_{n+1}^{i}
$$

Using the equation (3) we have that

$$
\begin{aligned}
\sum_{i=1}^{k} f_{1-i} f_{n+1}^{i}= & f_{0}\left(c_{1} f_{n}^{1}+c_{2} f_{n-1}^{1}+\cdots+c_{k} f_{n-k+1}^{1}\right) \\
& +f_{-1}\left(c_{1} f_{n}^{2}+c_{2} f_{n-1}^{2}+\cdots+c_{k} f_{n-k+1}^{2}\right) \\
& \vdots \\
& +f_{1-k}\left(c_{1} f_{n}^{k}+c_{2} f_{n-1}^{k}+\cdots+c_{k} f_{n-k+1}^{k}\right) \\
= & c_{1} \sum_{i=1}^{k} f_{1-i} f_{n}^{i}+c_{2} \sum_{i=1}^{k} f_{1-i} f_{n-1}^{i}+\cdots+c_{k} \sum_{i=1}^{k} f_{1-i} f_{n-k+1}^{i} .
\end{aligned}
$$

Then from the induction's hypothesis

$$
\sum_{i=1}^{k} f_{1-i} f_{n+1}^{i}=c_{1} f_{n}+c_{2} f_{n-1}+\cdots+c_{k} f_{n-k+1}=f_{n+1}
$$

and by the induction's rule the Theorem is proved.

TheOREM 2.2. Let $k \geq 2, n \geq 1,1 \leq i \leq k$ be integers. Then

$$
\begin{aligned}
& f_{n}^{i}=\sum_{t=0}^{k-i} \sum_{\substack{\alpha_{1}, \alpha_{2}, \ldots, \alpha_{k} \\
\alpha_{1}+2 \alpha_{2}+\cdots+k \alpha_{k}=n-t-i}} c_{i+t} \cdot c_{1}^{\alpha_{1}} \cdot c_{2}^{\alpha_{2}} \cdots c_{k}^{\alpha_{k}}\left(\begin{array}{c}
\alpha_{1}+\cdots+\alpha_{k} \\
\alpha_{1}, \ldots, \alpha_{k}
\end{array}\right) \\
& \text { for } c_{i}>0, i \in\{1, \ldots, k\}, \\
& f_{n}^{i}=\sum_{\substack{0 \leq t \leq k-i \\
c_{i+t}>0}} \sum_{\substack{\alpha_{1}, \alpha_{2}, \ldots, \alpha_{k} \\
\alpha_{1}+2 \alpha_{2}+\cdots+k \alpha_{k}=n-t-i}} c_{i+t} \cdot \prod_{c_{i}>0} c_{i}^{\alpha_{i}} \cdot\left(\begin{array}{c}
\alpha_{1}+\cdots+\alpha_{k} \\
\alpha_{1}, \ldots, \alpha_{k}
\end{array}\right) \\
& \text { if } n=\sum_{j=1}^{k} \alpha_{j} \cdot j, \text { for } c_{i} \geq 0, i \in\{1, \ldots, k\} \\
& \text { where } \alpha_{j}=0 \text { if } c_{j}=0 \text { or } \alpha_{j} \geq 0 \text { if } c_{j}>0 .
\end{aligned}
$$


Proof. The companion matrix $A$ of the sequence $\left\{f_{n}^{i}\right\}$ can be considered as an adjacency matrix of a special multidigraph. For convenience, lets consider a multidigraph $D$ defined by $A^{T}$ presented on the Fig. 1, where $c_{i}$ denotes the multiplicity of the $\operatorname{arc} v_{i} v_{1}$.

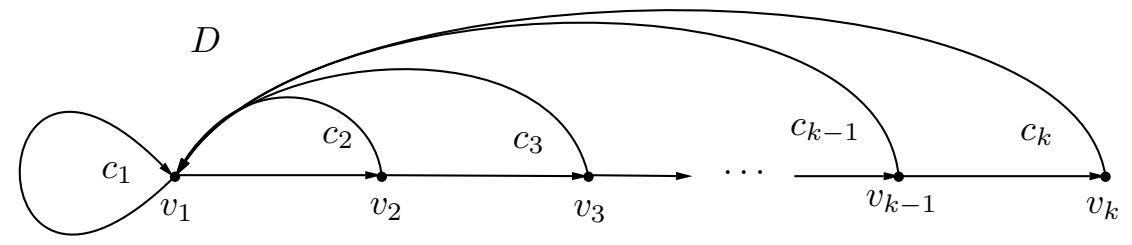

Figure 1. Digraph $D$.

Elements of the matrix $\left(A^{n}\right)^{T}=\left(A^{T}\right)^{n}$ give the number of all different paths of the length $n$ between corresponding vertices in the digraph $D$. For a fixed $1 \leq i \leq k$, the element $a_{i, 1}$ of the matrix $\left(A^{n}\right)^{T}$ is equal to the total number of different paths from $v_{i}$ to $v_{1}$. By (2), it holds that $a_{i, 1}=f_{n}^{i}$. Each such path consists of a path $v_{i}-\cdots-v_{i+t}-v_{1}, t \in\{0, \ldots k-i\}$, of the length $t+1$ and the finite sequence $\mathcal{C}$ of elementary cycles in random order. Cycles have the form $C_{i}=v_{1}-v_{2}-\cdots-v_{i}-v_{1}$ and lengths $i, i \in\{1,2, \ldots, k\}$. Suppose that the path consists of $\alpha_{i}$-times cycle $C_{i}$. Clearly, $\alpha_{i} \geq 0, i \in\{1,2, \ldots, k\}$. Then the length of this path can be written as $1 \alpha_{1}+2 \alpha_{2}+\cdots+k \alpha_{k}+t+1=n$ and by rewriting $1 \alpha_{1}+2 \alpha_{2}+\cdots+k \alpha_{k}=n-t-1$. To calculate the number of such paths observe that the arc $v_{i+t} v_{1}$ can be chosen in $c_{i+t}$ ways. The remaining part of this path consists of $\alpha_{1}+\alpha_{2}+\cdots+\alpha_{k}$ cycles $C_{i}, i \in\{1,2, \ldots, k\}$. The position of the cycle $C_{1}$ in the sequence $\mathcal{C}$ can be chosen in $\left(\begin{array}{c}\alpha_{1}+\alpha_{2}+\cdots+\alpha_{k} \\ \alpha_{1}\end{array}\right)$ ways, the position of the cycle $C_{2}$ in $\left(\begin{array}{c}\alpha_{2}+\cdots+\alpha_{k} \\ \alpha_{2}\end{array}\right)$ ways, and so on. Consequently, we have $\left(\begin{array}{c}\alpha_{1}+\alpha_{2}+\cdots+\alpha_{k} \\ \alpha_{1}\end{array}\right)\left(\begin{array}{c}\alpha_{2}+\cdots+\alpha_{k} \\ \alpha_{2}\end{array}\right) \cdots\left(\begin{array}{c}\alpha_{k} \\ \alpha_{k}\end{array}\right)$ possibilities that create a path of length $n-t-1$, which can be rewritten as a multinomial coefficient $\left(\begin{array}{c}\alpha_{1}+\cdots+\alpha_{k} \\ \alpha_{1} \ldots, \alpha_{k}\end{array}\right)$.

Since the cycle $C_{i}, i \in\{1,2, \ldots, k\}$, which appears $\alpha_{i}$ times in the sequence $\mathcal{C}$, can go through one of $c_{i}$ multiple arcs $v_{i} v_{1}$, by the multiplicity of it the number of sequences has to be multiplied by $c_{1}^{\alpha_{1}} \cdot c_{2}^{\alpha_{2}} \cdots c_{k}^{\alpha_{k}}$.

Summing over all possible collections $\alpha_{1}, \alpha_{2}, \ldots, \alpha_{k}$ satisfying the equality $1 \alpha_{1}+2 \alpha_{2}+\cdots+k \alpha_{k}=n-t-1$, we obtain that the number of sequences $\mathcal{C}$ is equal to

$$
\sum_{t=0}^{k-i} \sum_{\begin{array}{c}
\alpha_{1}, \alpha_{2}, \ldots, \alpha_{k} \\
\alpha_{1}+2 \alpha_{2}+\cdots+k \alpha_{k}=n-t-1
\end{array}} c_{i+t} \cdot c_{1}^{\alpha_{1}} \cdot c_{2}^{\alpha_{2}} \cdots c_{k}^{\alpha_{k}}\left(\begin{array}{c}
\alpha_{1}+\cdots+\alpha_{k} \\
\alpha_{1}, \ldots, \alpha_{k}
\end{array}\right)=f_{n}^{i} .
$$

To prove (5), observe that if there is $1 \leq i \leq k$ such that $c_{i}=0$ in (3), then the cycle $C_{i}$ does not exist in the path from $v_{i}$ to $v_{1}$ in the digraph $D$. Consequently $\alpha_{i}=0$ and we put $c_{i}^{\alpha_{i}}=1$ in the formula (4). Moreover we can omit terms which are equal to zero. Then the formula (5) follows. 


\section{IWONA WŁOCH - ANDRZEJ WŁOCH}

Using Theorem 2.1 and Theorem 2.2 we obtain

Corollary 2.3. Let $k \geq 2, n \geq 1,1 \leq i \leq k$ be integers. Then

$$
\begin{gathered}
f_{n}=\sum_{i=1}^{k} \sum_{t=0}^{k-i} \sum_{\substack{\alpha_{1}, \alpha_{2}, \ldots, \alpha_{k} \\
\alpha_{1}+2 \alpha_{2}+\cdots+k \alpha_{k}=n-t-1}} f_{i} \cdot c_{i+t} \cdot c_{1}^{\alpha_{1}} \cdot c_{2}^{\alpha_{2}} \cdots c_{k}^{\alpha_{k}}\left(\begin{array}{c}
\alpha_{1}+\cdots+\alpha_{k} \\
\alpha_{1}, \ldots, \alpha_{k}
\end{array}\right) \\
f_{n}=\sum_{\substack{1 \leq i \leq k \\
f_{i}>0}} \sum_{\substack{0 \leq t \leq k-i \\
c_{i+t}>0}} \sum_{\substack{\alpha_{1}, \alpha_{2}, \ldots, \alpha_{k} \\
\alpha_{1}+2 \alpha_{2}+\cdots+k \alpha_{k}=n-t-1}} f_{i} \cdot c_{i+t} \cdot \prod_{c_{i}>0}\left[c_{i}^{\alpha_{i}}\left(\begin{array}{c}
\alpha_{i}+\cdots+\alpha_{k} \\
\alpha_{i}
\end{array}\right)\right] \\
\text { if } n=\sum_{j=1}^{k} \alpha_{j} \cdot j, \quad \text { where } c_{i} \geq 0, i \in\{1, \ldots, k\} \\
\text { wher } \alpha_{j}=0 \quad \text { if } c_{j}=0 \quad \text { or } \alpha_{j} \geq 0 \text { if } c_{j}>0 .
\end{gathered}
$$

\begin{tabular}{|c|c|c|c|c|c|c|c|c|c|}
\hline 1 & 0 & 0 & 0 & 0 & 0 & 0 & 0 & 0 & 0 \\
\hline 1 & 1 & 0 & 0 & 0 & 0 & 0 & 0 & 0 & 0 \\
\hline 1 & 2 & 1 & 0 & 0 & 0 & 0 & 0 & 0 & 0 \\
\hline 1 & 3 & 3 & 1 & 0 & 0 & 0 & 0 & 0 & 0 \\
\hline 1 & 4 & 6 & 4 & 1 & 0 & 0 & 0 & 0 & 0 \\
\hline 1 & 5 & 10 & 10 & 5 & 1 & 0 & 0 & 0 & 0 \\
\hline 1 & 6 & 15 & 20 & 15 & 6 & 1 & 0 & 0 & 0 \\
\hline 1 & 7 & 21 & 35 & 35 & 21 & 7 & 1 & 0 & 0 \\
\hline 1 & 8 & 28 & 56 & 70 & 56 & 28 & 8 & 1 & 0 \\
\hline 1 & 9 & 36 & 84 & 126 & 126 & 84 & 36 & 9 & 1 \\
\hline 1 & 10 & 45 & 120 & 210 & 252 & 210 & 120 & 45 & 10 \\
\hline
\end{tabular}

It is well known that classical Fibonacci numbers can be calculated from the Pascal's triangle as a sum of binomials on special diagonals and this rule is described by the formula 1 . The same sums can be obtained from the Pascal's triangle using staircase method, see Fig. 2. The step has a height 1 and length 1.

FIGURE 2. Stairs method of calculating Fibonacci numbers from the Pascal's triangle.

The Corollary 2.3 gives direct formula for an arbitrary sequence defined by $k$ th order linear recurrence. We use this formula as a tool for determining other binomial formulas for these sequences. It also gives a possibility to discover graphical rules (called staircase method) for calculating these numbers from the Pascal's triangle. 


\section{ON SOME MULTINOMIAL SUMS RELATED TO THE FIBONACCI TYPE NUMBERS}

For explanation, consider as an example Fibonacci numbers and $n=11$. Then from (7) we have that

$F_{11}=\left(\begin{array}{c}10 \\ 0\end{array}\right)+\left(\begin{array}{l}9 \\ 0\end{array}\right)+\left(\begin{array}{l}9 \\ 1\end{array}\right)+\left(\begin{array}{l}8 \\ 1\end{array}\right)+\left(\begin{array}{l}8 \\ 2\end{array}\right)+\left(\begin{array}{l}7 \\ 2\end{array}\right)+\left(\begin{array}{l}7 \\ 3\end{array}\right)+\left(\begin{array}{l}6 \\ 3\end{array}\right)+\left(\begin{array}{l}6 \\ 4\end{array}\right)+\left(\begin{array}{l}5 \\ 4\end{array}\right)+\left(\begin{array}{l}5 \\ 5\end{array}\right)$.

These binomials marked on the Pascal's triangle give simple and elegant rule (stairs) of calculating $F_{11}$, see Fig. 3 . We can generalize this rule by extending

\begin{tabular}{|c|c|c|c|c|c|c|c|c|c|c|}
\hline 1 & 0 & 0 & 0 & 0 & 0 & 0 & 0 & 0 & 0 & 0 \\
\hline 1 & 1 & 0 & 0 & 0 & 0 & 0 & 0 & 0 & 0 & 0 \\
\hline 1 & 2 & 1 & 0 & 0 & 0 & 0 & 0 & 0 & 0 & 0 \\
\hline 1 & 3 & 3 & 1 & 0 & 0 & 0 & 0 & 0 & 0 & 0 \\
\hline 1 & 4 & 6 & 4 & 1 & 0 & 0 & 0 & 0 & 0 & 0 \\
\hline 1 & 5 & 10 & 10 & 5 & 1 & 0 & 0 & 0 & 0 & 0 \\
\hline 1 & 6 & 15 & 20 & 15 & 6 & 1 & 0 & 0 & 0 & 0 \\
\hline 1 & 7 & 21 & 35 & 35 & 21 & 7 & 1 & 0 & 0 & 0 \\
\hline 1 & 8 & 28 & 56 & 70 & 56 & 28 & 8 & 1 & 0 & 0 \\
\hline 1 & 9 & 36 & 84 & 126 & 126 & 84 & 36 & 9 & 1 & 0 \\
\hline 1 & 10 & 45 & 120 & 210 & 252 & 210 & 120 & 45 & 10 & 1 \\
\hline
\end{tabular}

FiguRE 3. Stairs method of calculating $F_{11}$ from the Pascal's triangle.

staircases in two directions. Moving such infinite staircases downwards, we can calculate the next Fibonacci number.

Based on such graphical rule, we can give a new formula for Fibonacci numbers

$$
F_{n}=\sum_{i=0}^{\left\lfloor\frac{n}{2}\right\rfloor} \sum_{j=0}^{1}\left(\begin{array}{c}
n-1-i-j \\
i
\end{array}\right)
$$

We have two binomials adjacent in a row on each step of a staircase. Using the basic formula

$$
\left(\begin{array}{l}
n \\
k
\end{array}\right)+\left(\begin{array}{c}
n \\
k+1
\end{array}\right)=\left(\begin{array}{l}
n+1 \\
k+1
\end{array}\right)
$$

we immediately obtain that

$$
F_{n}=\sum_{i=0}^{\left\lfloor\frac{n}{2}\right\rfloor}\left(\begin{array}{c}
n-i \\
i
\end{array}\right)
$$

Let us consider now Padovan numbers defined by $P v(n)=P v(n-2)+P v(n-3) \quad$ for $n \geq 0$ with $P v(-2)=P v(-1)=P v(0)=1$. 


\section{IWONA WŁOCH - ANDRZEJ WŁOCH}

According to Theorem 2.1 we have that

$$
P v(n)=\sum_{i=1}^{3} P v(1-i) P v^{i}(n)=P v^{1}(n)+P v^{2}(n)+P v^{3}(n) .
$$

For example, let $n=11$. By (77) we have that

$$
\begin{aligned}
& P v^{1}(11)=\left(\begin{array}{l}
4 \\
0
\end{array}\right)+\left(\begin{array}{l}
4 \\
1
\end{array}\right)+\left(\begin{array}{l}
3 \\
2
\end{array}\right)+\left(\begin{array}{l}
3 \\
3
\end{array}\right) \quad \text { (solid line on the Fig. 4), } \\
& P v^{2}(11)=\left(\begin{array}{l}
5 \\
0
\end{array}\right)+\left(\begin{array}{l}
4 \\
1
\end{array}\right)+\left(\begin{array}{l}
4 \\
2
\end{array}\right)+\left(\begin{array}{l}
3 \\
3
\end{array}\right) \quad \text { (dashed line), } \\
& P v^{3}(11)=\left(\begin{array}{l}
4 \\
0
\end{array}\right)+\left(\begin{array}{l}
3 \\
1
\end{array}\right)+\left(\begin{array}{l}
3 \\
2
\end{array}\right) \quad \text { (dotted line). }
\end{aligned}
$$

\begin{tabular}{|c|c|c|c|c|c|c|c|c|c|c|}
\hline 1 & 0 & 0 & 0 & 0 & 0 & 0 & 0 & 0 & $\underline{0}$ & 0 \\
\hline 1 & 1 & 0 & 0 & 0 & 0 & 0 & 0 & 0 & $\overline{0}$ & 0 \\
\hline 1 & 2 & 1 & 0 & 0 & 0 & 0 & 0 & 0 & 0 & 0 \\
\hline 1 & 3 & 3 & 1 & 0 & 0 & 0 & 0 & 0 & 0 & 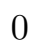 \\
\hline 1 & 4 & 6 & 4 & 1 & 0 & 0 & 0 & 0 & 0 & 0 \\
\hline $1_{-}$ & 5 & 10 & 10 & 5 & 1 & 0 & 0 & 0 & 0 & 0 \\
\hline 1 & 6 & 15 & 20 & 15 & 6 & 1 & 0 & 0 & 0 & U \\
\hline 1 & 7 & 21 & 35 & 35 & 21 & 7 & 1 & 0 & 0 & 0 \\
\hline 1 & 8 & 28 & 56 & 70 & 56 & 28 & 8 & 1 & 0 & 0 \\
\hline 1 & 9 & 36 & 84 & 126 & 126 & 84 & 36 & 9 & 1 & 0 \\
\hline 1 & 10 & 45 & 120 & 210 & 252 & 210 & 120 & 45 & 10 & 1 \\
\hline
\end{tabular}

These binomials marked in the Pascal's triangle form stairs. By extending them we obtain a rule for calculating $P v^{1}(n), P v^{2}(n), P v^{3}(n)$, see the Fig. 4.

Figure 4. Calculating $P v^{1}(11), P v^{2}(11), P v^{3}(11)$ by (5).

Consequently,

Moreover,

$$
P v^{1}(n)=\sum_{i=0}^{\left\lfloor\frac{n-4}{2}\right\rfloor}\left(\begin{array}{c}
\left\lfloor\frac{n-2-i}{2}\right\rfloor \\
i
\end{array}\right)
$$

$$
P v^{2}(n)=P v^{1}(n+1) \text { and } P v^{3}(n)=P v^{1}(n-1) .
$$

Using (9) in each step for adjacent binomials we obtain a new pattern for calculating these numbers, see Fig. 5. This leads to

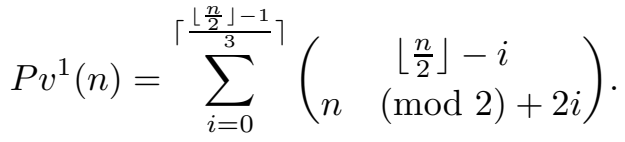


Analogously,

$$
P v^{2}(n)=P v^{1}(n+1) \text { and } P v^{3}(n)=P v^{1}(n-1) .
$$

\begin{tabular}{|c|c|c|c|c|c|c|c|c|c|c|}
\hline 1 & 0 & 0 & 0 & 0 & 0 & 0 & 0 & 0 & 0 & 0 \\
\hline 1 & 1 & 0 & 0 & 0 & 0 & 0 & 0 & 0 & 0 & $\ddot{0}$ \\
\hline 1 & 2 & 1 & 0 & 0 & 0 & 0 & 0 & 0 & 0 & $\overline{0}$ \\
\hline 1 & 3 & 3 & 1 & 0 & 0 & $\ddot{0}$ & 0 & $\overline{0}$ & 0 & 0 \\
\hline 1 & 4 & 6 & 4 & 1 & 0 & $\overline{0}$ & 0 & 0 & 0 & 0 \\
\hline 1 & 5 & 10 & 10 & $\overline{5}$ & 1 & 0 & 0 & 0 & 0 & 0 \\
\hline 1 & 6 & $\overline{15}$ & 20 & 15 & 6 & 1 & 0 & 0 & 0 & 0 \\
\hline$\overline{1}$ & 7 & 21 & 35 & 35 & 21 & 7 & 1 & 0 & 0 & 0 \\
\hline 1 & 8 & 28 & 56 & 70 & 56 & 28 & 8 & 1 & 0 & 0 \\
\hline 1 & 9 & 36 & 84 & 126 & 126 & 84 & 36 & 9 & 1 & 0 \\
\hline 1 & 10 & 45 & 120 & 210 & 252 & 210 & 120 & 45 & 10 & 1 \\
\hline
\end{tabular}

Figure 5. Calculating $P v^{1}(11), P v^{2}(11), P v^{3}(11)$ by (5) and (9).

Using twice the formula (9) we obtain

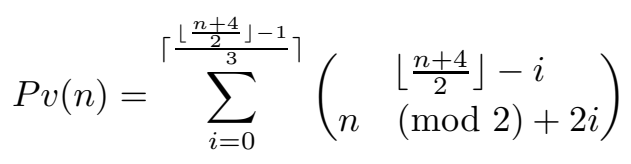

and the corresponding stairs are presented on the Fig. 6.

$$
\begin{array}{lllllllllll}
1 & 0 & 0 & 0 & 0 & 0 & 0 & 0 & 0 & 0 & 0 \\
1 & 1 & 0 & 0 & 0 & 0 & 0 & 0 & 0 & 0 & 0 \\
1 & 2 & 1 & 0 & 0 & 0 & 0 & 0 & 0 & 0 & 0 \\
1 & 3 & 3 & 1 & 0 & 0 & 0 & 0 & 0 & 0 & 0 \\
1 & 4 & 6 & 4 & 1 & 0 & 0 & 0 & 0 & 0 & 0 \\
1 & 5 & 10 & 10 & 5 & 1 & 0 & 0 & 0 & 0 & 0 \\
1 & 6 & 15 & 20 & 15 & 6 & 1 & 0 & 0 & 0 & 0 \\
1 & 7 & 21 & 35 & 35 & 21 & 7 & 1 & 0 & 0 & 0 \\
1 & 8 & 28 & 56 & 70 & 56 & 28 & 8 & 1 & 0 & 0 \\
1 & 9 & 36 & 84 & 126 & 126 & 84 & 36 & 9 & 1 & 0 \\
1 & 10 & 45 & 120 & 210 & 252 & 210 & 120 & 45 & 10 & 1
\end{array}
$$

Figure 6 . Calculating $P v(11)$ by (11). 


\title{
IWONA WŁOCH - ANDRZEJ WŁOCH
}

\section{REFERENCES}

[1] BEDNARZ, U.-WŁOCH, I.-WOŁOWIEC-MUSIAE, M.: Total graph interpretation of the number of the Fibonacci type, J. Appl. Math. (2015), Article ID 837917, 7 pp.

[2] ER, M. C.: Sums of Fibonacci numbers by matrix methods, Fibonacci Quart. 22 (1984), no. 3, 204-207.

[3] FALCÓN SANTANA, S.-DÍAZ-BARRERO, J. L.: Some properties of sums involving Pell numbers. Missouri J. Math. Sci. 18 (2006), no. 1, 33-40.

[4] HORADAM, A. F.: Pell identities, Fibonacci Quart. 9 (1971), no. 3, 245-252, 263.

[5] D. KALMAN: Generalized Fibonacci numbers by matrix methods, Fibonacci Quart. 20 (1982), no. 1, 73-76.

[6] KILIÇ, E.-PRODINGER, H.: Some double binomial sums related to Fibonacci, Pell and generalized order-k Fibonacci numbers, Rocky Mountain J. Math. 43 (2013), no. 3, 975-987. https://projecteuclid.org/euclid.rmjm/1375361983

[7] KOSHY, T.: Fibonacci, Lucas, and Pell numbers, and Pascal's triangle, Appl. Probab. Trust, (2011), 125-132.

[8] LEE, G.-Y.-LEE, S.-G.-KIM, J.-S.-SHIN, H. K.: The Binet formula and representations of k-generalized Fibonacci numbers, Fibonacci Quart. 39 (2001), no. (2) 158-164.

[9] MILES, E. P. JR.: Generalized Fibonacci numbers and associated matrices, Amer. Math. Monthly, 67 (1960), 745-752.

[10] SLOANE, N.J.A.: (an editor). The On-Line Encyclopedia of Integer Sequences, https://oeis.org (published electronically).

Received March 31, 2020

\author{
Iwona Wtoch \\ Department of Discrete Mathematics \\ The Faculty of Mathematics and Applied Physics \\ Rzeszow University of Technology \\ al. Powstancow Warszawy 8 \\ 35-959 Rzeszow \\ POLAND \\ E-mail: iwloch@prz.edu.pl \\ Andrzej Wtoch \\ Department of Mathematical Modeling \\ The Faculty of Mathematics and Applied Physics \\ Rzeszow University of Technology \\ al. Powstancow Warszawy 8 \\ 35-959 Rzeszow \\ POLAND \\ E-mail: awloch@prz.edu.pl
}

